\title{
Estudio crítico de la revista "Voprosy Kurortologii Fizioterpii i Lechbenoi Fiziches koi Kultury" a traves de los trabajos sobre peloides publicados en los últimos 5 años
}

\author{
Narciso GRAÑA ${ }^{(1)}$, José Luís ARRANZ ${ }^{(1)}$, \\ Margarita GARCÍA-SÁNCHEZ ${ }^{(1)}$, M $^{\mathrm{a}}$ Elisa PÁRRAGA DE LAS MARINAS ${ }^{(1)}$ \\ ${ }^{(1)}$ Escuela Profesional de Hidrología Médica e Hidroterapia. Facultad de Medicina. \\ Universidad Complutense de Madrid. Plaza Ramón y Cajal s/n, 28040 Madrid (España). \\ hidromed@med.ucm.es
}

Recibido: $30-12-10$

Aceptado: 12-04-11

\section{Resumen}

En una publicación reciente de Hidrología Médica nos llamó la atención que se destacara que, en la base de datos MEDLINE, la publicación científica con mayor número de trabajos publicados sobre Hidrología Médica sea la revista rusa "Voprosy Kurortologii, Fizioterapii i Lechbenoi Fizicheskoi Kultury". Por ello se realizó una recopilación de los artículos publicados sobre peloides en los últimos 5 años (periodo 2005-2010) con el objetivo de realizar un estudio crítico de los mismos, mediante un análisis multifactorial de su calidad científica. De los 21 artículos encontrados 10 de ellos cumplieron con los criterios de selección.

Palabras claves: Vopr Kurortol Fizioter Lech Kult; Barroterapia; Peloterapia

Critical analysis of journal

"Voprosy Kurortologii Fizioterpii i Lechbenoi Fizicheskoi Kultury" by the papers about peloids publisheds in the last 5 years

\begin{abstract}
In a recent publication in Medical Hidrology it drew our attention to the fact that, in MEDLINE data base, the scientific publication with more published articles about Medical Hidrology is the Russian journal "Voprosy Kurortologii, Fizioterapii i Lechbenoi Fizicheskoi Kultury". It is for this reason that we recompilated the published articles about peloids in the last 5 years (2005-2010 period), with the objective to carry out a critical study of them, using a multifactor analysis of the scientific quality. We found 21 articles, and 10 of them achieve the selction criteria.
\end{abstract}

Key words: Vopr Kurortol Fizioter Lech Kult, Mud therapy, Pelotherapy 
REFERENCIA NORMALIZADA

Graña N, Arranz JL, García-Sánchez M, Párraga E. Estudio crítico de la revista "Voprosy Kurortologii Fizioterpii i Lechbenoi Fiziches koi Kultury" a traves de los trabajos sobre peloides publicados en los últimos 5 años. (Critical analysis of journal "Voprosy Kurortologii Fizioterpii i Lechbenoi Fizicheskoi Kultury" by the papers about peloids publisheds in the last 5 years). Anal Hidrol Med, 2011, vol. 4, $117-127$

\section{INTRODUCCIÓN}

En un trabajo recientemente publicado sobre investigación en Hidrología Médi$\mathrm{ca}^{1}$, nos llamó poderosamente la atención que se destacara, como en la base de datos de artículos biomédicos MEDLINE, la publicación científica con mayor número de trabajos publicados sobre Hidrología Médica en los últimos años era rusa, concretamente la revista "Voprosy Kurortologii, Fizioterapii i Lechbenoi Fizicheskoi Kultury" (VKLF).

El objetivo de presente trabajos es realizar un estudio crítico sobre los trabajos de peloides y/ó peloterapia publicados en la citada revista durante los últimos 5 años, a través del análisis multifactorial de la calidad de la investigación publicada.

\section{MATERIAL Y MÉTODOS}

\section{Material}

Para ello hemos realizado una búsqueda en PubMed cuyos límites fueron los siguientes:

- Russian

- Vopr Kurortol Fizioter Lech Kult AND Mud therapy AND Pelotherapy

- With abstracts

- Published the last 5 years

Se encontraron 23 artículos indexados. De los cuales diez, correspondía a referencias de otras publicaciones; dos, no presentaban autor/es; uno, carecía de contenido y, sólo diez cumplieron con los siguientes criterios: ser publicados por la revista VKLF, contenido clínico y autores relacionados en la busqueda. para ser analizados (Figura 1).

Posteriormente solicitamos los trabajos al Servicio Interbibliotecario de la Facultad de Medicina de la Universidad Complutense de Madrid, (la biblioteca intentó localizar los artículos en otras bibliotecas españolas, sin éxito, por lo que se buscaron los artículos en otras bibliotecas europeas concertadas consiguiéndose al final en Alemania), los artículos fueron dados a dos traductores de forma aleatoria y a 
doble ciego para evitar intereses personales procediéndose a su traducción y análisis.

Figura 1 - Trabajos encontrados

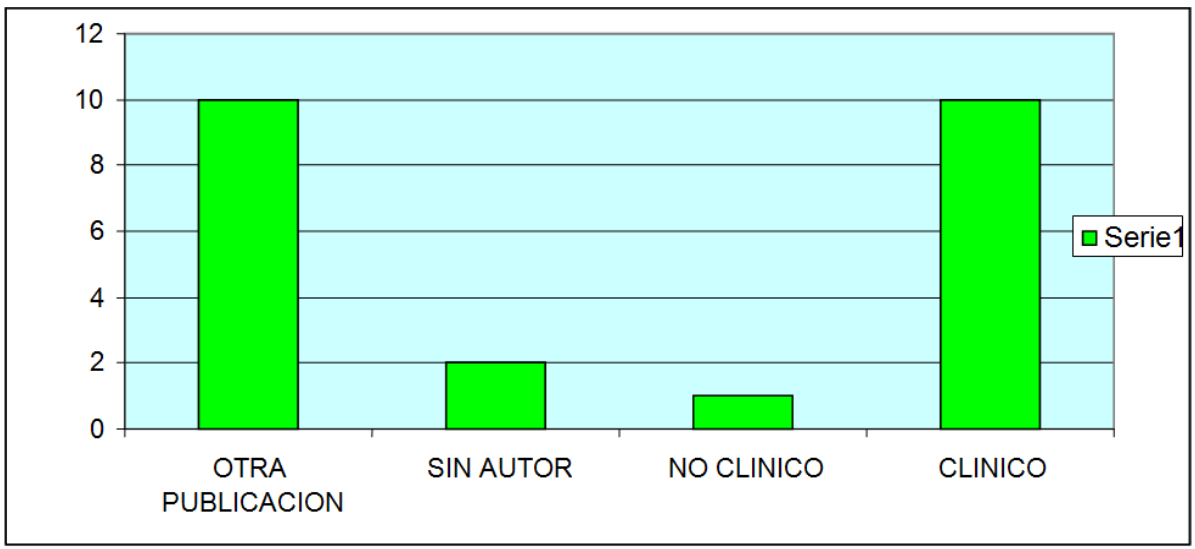

\section{Método}

Para el análisis de los artículos hemos utilizado las directrices de revisión RATS modificadas por BioMed Central ${ }^{2}$ a partir del trabajo de Jocalyn Clark (Clark JP: How to peer review a qualitative manuscript. In Peer Review in Health Sciences. Second edition. Edited by Godlee F, Jefferson T. London: BMJ Books; 2003:219235).

Una vez hecha una primera aproximación, dimos puntuación a los distintos parámetros introducidos en el RATS, que modificamos ligeramente para ajustarnos a los distintos tipos de artículos publicados. Valoramos de 1 a 3 puntos cada item del RATS: 1 punto para los no adecuados, 2 puntos para los adecuados pero con fallos y 3 puntos para los totalmente adecuados (Tabla 1).

Por otra parte, también hemos valorado los niveles de evidencia científica ${ }^{3}$, aplicando las tablas de calidad elaboradas por la Agencia de Evaluación de Tecnología Médica $^{4}$ (AETM) (Tabla 2) y los criterios del Centro para la Medicina basada en la evidencia (CEBM) de la Universidad de Oxfor ${ }^{5}$ (Tabla 3)

No se necesita ningún manejo estadístico, debido a que los datos recogidos proceden de una lista previa y no hay diferencias entre artículos. 
Tabla 1 - Puntuación del RATS

\begin{tabular}{|l|l|l|}
\hline \multicolumn{1}{|c|}{ PUNTUACION RATS } & & \\
\hline R: Relevancia del estudio & 1 & NO ADECUADO \\
\hline estudio & 2 & ADECUADO CON FALLOS \\
\hline & 3 & TOTALMENTE ADECUADO \\
\hline A: Apropiado o propiedad & 1 & NO ADECUADO \\
\hline del estudio & 2 & ADECUADO CON FALLOS \\
\hline & 3 & TOTALMENTE ADECUADO \\
\hline T: Transparencia de & 1 & NO ADECUADO \\
\hline los procedimientos & 2 & ADECUADO CON FALLOS \\
\hline & 3 & TOTALMENTE ADECUADO \\
\hline S: Sentido (concordancia) & 1 & NO ADECUADO \\
\hline del análisis & 2 & ADECUADO CON FALLOS \\
\hline & 3 & TOTALMENTE ADECUADO \\
\hline
\end{tabular}

Tabla 2 - Niveles de calidad de evidencia cientifica del AETM

\begin{tabular}{|l|l|l|}
\hline $\begin{array}{l}\text { NIVELES DE CALIDAD DE } \\
\text { EVIDENCIA CIENTIFICA }\end{array}$ & $\begin{array}{l}\text { FUERZA DE LA } \\
\text { EVIDENCIA }\end{array}$ & TIPO DE DISEÑO \\
\hline I & Adecuada & Meta análiisis \\
\hline II & Adecuada & ECA muestra grande \\
\hline III & Buena a regular & ECA muestra pequeña \\
\hline IV & Buena a regular & $\begin{array}{l}\text { Ensayo prospectivo } \\
\text { controlado }\end{array}$ \\
\hline V & Regular & $\begin{array}{l}\text { Ensayo retrospectivo } \\
\text { controlado }\end{array}$ \\
\hline VI & Regular & Estudios de cohorte \\
\hline VII & Regular & E casos control \\
\hline VIII & Pobre & $\begin{array}{l}\text { Series clinicas, consensos } \\
\text { descriptivos }\end{array}$ \\
\hline IX & Pobre & Casos únicos \\
\hline
\end{tabular}


Tabla 3 - Grados de recomendación científica CEMB

\begin{tabular}{|l|l|c|}
\hline $\begin{array}{l}\text { GRADOS DE } \\
\text { RECOMENDACIÓN }\end{array}$ & \\
\hline A & estudios de nivel 1 & $\begin{array}{c}\text { Extremadamente } \\
\text { recomendable }\end{array}$ \\
\hline B & Estudios de nivel 2-3 & $\begin{array}{c}\text { Recomendación } \\
\text { favorable }\end{array}$ \\
\hline C & Estudios de nivel 4 & $\begin{array}{c}\text { Recomendación favorable no } \\
\text { concluyente }\end{array}$ \\
\hline D & Estudios de nivel 5 & $\begin{array}{c}\text { Ni se recomienda ni se des- } \\
\text { aprueba }\end{array}$ \\
\hline
\end{tabular}

\section{RESULTADOS}

Los trabajos que cumplieron los criterios anteriormente expuestos son referenciados por orden cronológico, acompañados de la traducción de sus resúmenes:

1. Nasirov MIa, Efendieva FM, Ismaǔlova DA. [The influence of peloids from volcanic deposits in Azerbaijan on the dynamics of sugar content in blood and urine and the wound healing in patients at the early stages of diabetic gangrene of the lower extremities] Vopr Kurortol Fizioter Lech Fiz Kult 2009 NovDec;(6):42-3.

[Influencia de los peloides de depósitos volcánicos en Azerbaiyan en la dinámica del contenido de glucosa en sangre y orina y en la curación de ulceras en estadios tempranos en pacientes con gangrena diabética de las extremidades inferiores]

Resumen

El tratamiento de pacientes diabéticos com lesiones necróticas de las extremidades inferiores requiere prolongadas sesiones de balneo-fisioterapia para normalizar las funciones de los centros nerviosos. Soluciones de fango volcánico (15-24 g/l) enriquecidos con componentes orgánicos e inorgánicos biológicamente activos de depósitos volcánicos de Azerbaiyan han sido usadas por primera vez para tratar a estos pacientes. Los peloides fueron aplicados en la región lumbar (situación de los nodos de los nervios simpáticos) y en las piernas, por debajo y alrededor de los sitios afectados. Se realizaron de 12 a 15 sesiones de peloterapia a $40^{\circ} \mathrm{C}$ durante 20 o 30 minutos. El tratamieto fue seguido de cura de la úlcera según procedimientos estandar y tratamiento antibiótico según antibiograma. Un total de 86 pacientes diabéticos con gangrena en las piernas fueron revisados con termografía vascular que revelo aumento de afluencia de sangre en las extremidades bajo el efecto de los peloi- 
des. La peloterapia dio como resultado la normalización de los niveles de glucosa en sangre y orina de $53(63 \%)$ de los pacientes. Simultaneamente la dosis de medicamentos disminuyó. La curación de la úlcera fue completa en la mayoría de los pacientes (86\%), al final de la balneo-fisioterapia cuando se empezó a desarrollar tejido de granulación y los signos de edema desaparecieron. No fue necesaria la amputación de ningún paciente.

2. Khodova TV, Shvedunova LN, Babina LM [A new method of peloidotherapy for the rehabilitation of children living in environmentally unfavorable regions]. Vopr Kurortol Fizioter Lech Fiz Kult 2009 Jul-Aug;(4):48-9.

[Nuevo método de peloterapia para la rehabilitación de niños que viven en regiones de medioambiente hostil]

Resumen

Este artículo esta dedicado al problema de terapia de la rehabilitación en balnearios en niños con el Síndrome de desadaptación ecológica. Se describen aproximaciones originales a la eficacia del tratamiento con referencias a las diferentes modalidades terapéuticas. El estudio demuestra ventajas con la peloterapia actuando sobre las respuestas reflejas y adaptativas de los pacientes, por encima de los métodos tradicionales de aplicación de peloides.

3. Bykov AT, Konovalova MP, Khodasevich LS. [Magneto-peloidotherapy and hydrogen sulfide baths for the correction of dyslipidemia and immune inflammation in patients with ischemic heart disease during resort treatment] Vopr Kurortol Fizioter Lech Fiz Kult 2009 Jul-Aug;(4):17-9.

[Magneto-peloterapia y baños de agua sulfurada para la corrección de la dislipemia y la inflamación inmune en pacientes com enfermedad coronaria en balnearios]

Resumen

Un total de 55 pacientes con angina de esfuerzo (clase funcional I-II) fueron tratados con magneto-peloterapia y baños de agua sulfurada. La efectividad del tratamiento fue evaluada por el perfil lipidico (colesterol total, triglicéridos, HDL, LDL), índice de aterogenicidad, peroxidación lipídica, reactividad del sistema de defensa antioxidativo y características inmunes. Los resultados del estudio indican que la combinación de la magneto-peloterapia y los baños tienen efectos hipolipidémicos y reducen la peroxidación lipídica en ausencia de activación del sistema de defensa antioxidativo y la corrección del disbalance del sistema autoinmune. En su conjunto estos efectos disminuyen la severidad de la reacción del sistema inflamatorio y facilitan la remisión del proceso ateroesclerótico. 
4. Rassulova MA, Siziakova LA, Aurrapetova NS. [Effect of naphthalan and therapeutic mud applications on clinical and roentgenological characteristics in patients with protracted pneumonia] Vopr Kurortol Fizioter Lech Fiz Kult 2009 May-Jun;(3):16-9.

[Efectos de la aplicación de naftalán y fango terapeutico en pacientes con clínica y signos radiográficos en sujetos con neumonía previamente tratada]

Resumen

La influencia de la aplicación de naftalan y peloide en los parâmetros clínicos y radiológicos, función respiratória, características biomecánicas e inmunológicas del proceso inflamatório fueron estudiados en 82 pacientes con neumonía tratada previamente y comparados con los resultados de los tratamientos sin terapia física. La aplicación de naftalan y peloide reducen el proceso inflamatório, la permeabilidad de la via aérea y el estado de la mucosa bronquial. El naftalan produce mejores efectos con el fango.

5. Starichkov AA, Bondareva ZG. [Use of resort factors in Novosibirsk region in the treatment of digestion system diseases] Vopr Kurortol Fizioter Lech Fiz Kult 2007 Nov-Dec;(6):11-3.

[Uso de los recursos termales del balneario de Novosibirsk en el tratamiento de las enfermedades del aparato digestivo]

Resumen

El agua mineral carbonatada clorurada sódica tiene efectos procinéticos, reduce la litogenicidad de las sales biliares y disminuye el nivel de las glicoproteinas mejorando sus características reológicas. Los baños termales salinos disminuyen el $\mathrm{pH}$ estomacal, estimulan la secreción pancreática, normalizan la composición de la mucosa y activan los mecanismos adaptativos del organismo. La peloterapia modula la acidez del jugo gástrico mediante el efecto antielicobacteria y disminuyen la concentración de glicoproteinas en el intestino.

6. Shutov AA, Panasiuk IIa. [Efficacy of rehabilitation of patients with chronic primary low back pain at the spa Klyuchi using balneopelotherapy and transcranial electrostimulation] Vopr Kurortol Fizioter Lech Fiz Kult 2007 MarApr;(2):16-8.

Resumen

[Eficacia de la rehabilitacion en pacientes con lumbalgia primaria en el balneario Klyuchi usando balneopeloterapia y electroestimulacion transcraneal] 
La lumbalgia causada por patología vertebral esta presente en el $60-80 \%$ de la población adulta. Estudiamos la eficacia de los factores de los peloides del balneario de Klyuchi y la electroestimulación transcraneal en 60 pacientes con lumbalgia primaria. Los pacientes fueron divididos en dos grupos. Los 30 pacientes del grupo 1 recibieron recibieron peloterapia con fango sulfurado, 30 pacientes del grupo 2 recibieron la misma peloterapia añadiendo electroestimulación transcraneal. La disminución del dolor y el estatus psicovegetativo mejoró en ambos grupos pero el efecto fue más pronunciado en el grupo 2 .

7. Barnatskiı̌ VV. [Peloid therapy in various temperature modes in rehabilitation of patients with seronegative spondylarthritis] Vopr Kurortol Fizioter Lech Fiz Kult 2007 Mar-Apr;(2):7-12.

\section{[Peloterapia a distintas temperaturas en la rehabilitacion de pacientes con espondiloartritis seronegativa]}

Resumen

Quince pacientes con espondiloartritis anquilosante y 21 pacientes con artritis reactiva y enfermedad de Reiter recibieron tratamiento con peloide. Los pacientes con espondilitis y una lesión espinal prevalerte recibieron aplicaciones de fango $\left(38^{\circ}-40^{\circ}\right)$ y los que tenían una lesión en las articulaciones aplicaciones de fango frio $\left(22^{\circ}-24^{\circ}\right)$, aquellos libres de inflamación en las articulaciones y tejidos periarticulares recibieron barro a $28^{\circ}-40^{\circ} \mathrm{C}$. En la artritis reactiva y la enfermedad de Reiter las aplicaciones de barro a temperatura de $22-24^{\circ}$ están indicadas.

8. Gorbunov IuV, Subbotin SP, Shkliaev AE. [Efficacy of SMC-electrophoresis of peat mud in primary chronic duodenitis] Vopr Kurortol Fizioter Lech Fiz Kult 2006 Jul-Aug;(4):25-7.

\section{[Eficacia de la electroforesis sinusoidal modulada en barro de turba para la duodenitis crónica primaria]}

Resumen

La electroforesis sinusoidal modulada en barro de turba ha sido usada como monoterapia de la duodenitis crónica primaria en el balneario Uva. Un total de 65 pacientes con duodenitis crónica recibieron barro de turba con electroforesis diluida con agua destilada. El efecto fue seguido con fibrogastroduodenoscopia y biopsia de la mucosa duodenal con estudio de su estructura morfológica, producción de ácido, perfil hormonal, metabolismo colágeno. Cambios positivos fueron observados en los cambios clínicos, endoscópicos y morfológicos de la enfermedad los cuales son explicados por cambios favorables en el estatus hormonal y en la normalización del metabolismo del colágeno de la mucosa duodenal. 
9. Ibadova GD, Mamishev SN, Zavora MI, Sarian LA. [Combined use of hydrogen sulfide baths and galvanopeloid therapy with magnetolaser radiation in osteoarthrosis patients at a health resort] Vopr Kurortol Fizioter Lech Fiz Kult 2005 Jul-Aug;(4):23-5.

\section{[Uso combinado de baños de agua sulfurada y terapia con peloide galvanizado con radiación de magnetolaser en pacientes con osteo- artrosis en el medio termal]}

Resumen

El estudio incluye 174 pacientes con osteoartrosis en estadio radiológico I y II, y sinovitis reactiva leve o subclínica. Los pacientes recibieron tratamiento balneario incluyendo baños sulfurados, galvanopeloterapia con fango sulfurado de los depósitos de Imeretinsk por la técnica segmentaria local, junto con radiación con láser magnético en algunos casos. Se encontraron efectos analgésicos y antiinflamatorios que fueron mayores con la utilización consecutiva del laser y la peloterapia, mostradas por normalización de la función autonómica, mejora de la circulación periférica inhibición de la proteolisis debida a acciones adaptativas y antioxidantes.

10. Barnatskii VV. [Effects of radon and peloid therapy on functional condition and quality of life in patients with seronegative spondyloarthritis] Vopr Kurortol Fizioter Lech Fiz Kult. 2005 May-Jun;(3):26-30

\section{[Efectos del radón y peloterapia sobre las condiciones funcionales y la calidad de vida en pacientes con espondiloartrosis seronegativa]}

Resumen

Radón y peloterapia fue administrada a 88 pacientes con espondilitas seronegativa, de ellos 58 tenían espondiloartritis anquilosante (30 tenían efectos mas severos de la columna vertebral y 28 de las articulaciones periféricas), 30 tenían artritis y enfermedad de Reiter. Baños de agua radiactiva con Radón y aplicaciones de peloide a baja temperatura produjeron en todos los pacientes efectos analgésicos y antiinflamatorios, mejorando la condición funcional y calidad de vida, por ejemplo habilidades ocupacionales, cuidados personales, bienestar y libido.

Los temas de los artículos en función de las patologías tratadas y la especialidad médica relacionada fueron variados predominando la patología reumatológica con cuatro de los diez trabajos, las otras especialidades tratadas fueron digestivo con dos artículos y pediatría, endocrinología, patología cardiovascular y neumología con uno cada una. 
En la puntuación que hemos realizado según la escala RATS, el menor resultado que pueden alcanzar los artículos es de 3 y el más alto es de 12. En la revisión realizada se han puntuado 1 con 6 puntos, 2 con 7 puntos, 3 con 8 puntos, 3 con 9 puntos y 1 con 10 puntos (Figura 2).

Figura 2 - Puntuación obtenida según RATS

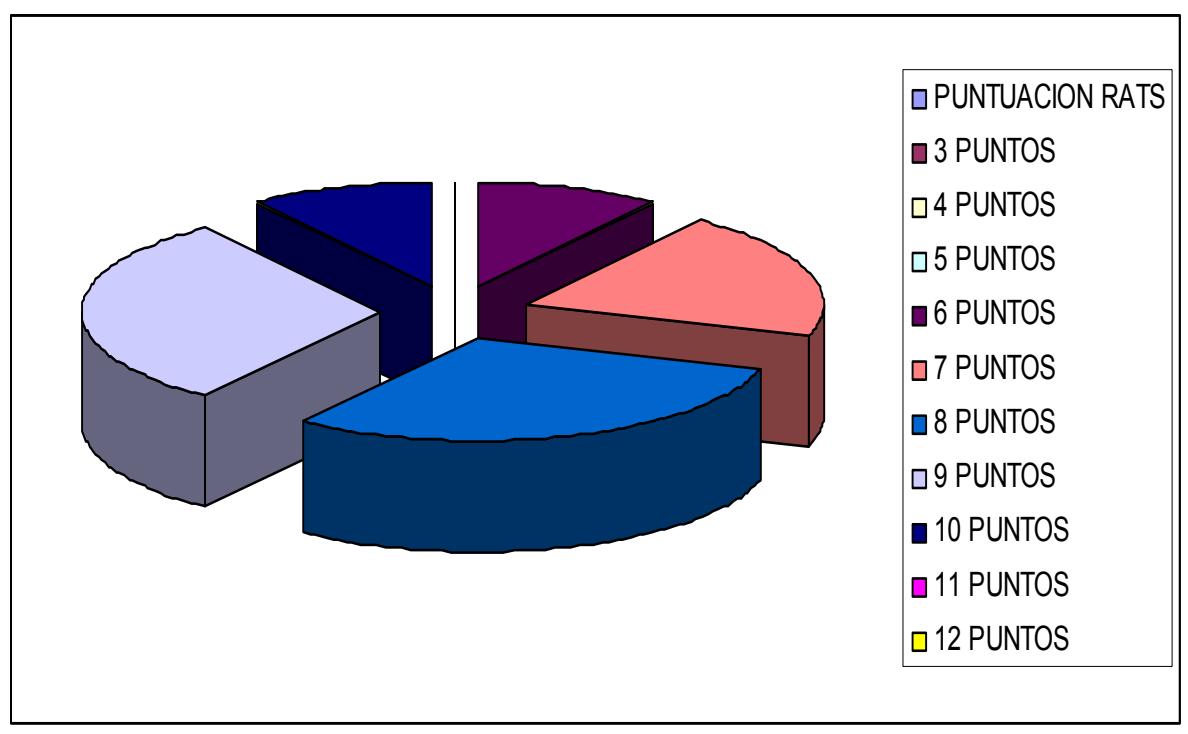

Las principales deficiencias halladas en los trabajos estudiados se encuentra en los ítems $\mathrm{T}$ (transparencia de los procedimientos) y $\mathrm{S}$ (sentido de los análisis realizados), sobre todo en la obtención de la muestra, el reclutamiento de la misma y la recolección de datos, así como en el tipo de análisis y en la interpretación estadística.

Tipo de trabajo. De los artículos analizados cuatro fueron ensayos clínicos, cinco casos control y uno descriptivo.

Según el grado de recomendación de la CEMB todos los artículos tienen una gran relevancia en sus contenidos con un grado de recomendación $\mathrm{B}$ y un nivel de evidencia de II a III.

Según los niveles de calidad en la evidencia científica de la AETM observamos que los artículos tienen un nivel de calidad que oscila entre III a VIII sobre IX. Cuatro tienen un nivel de III, cinco tienen un nivel de VII y uno de VIII. Lo que significa que los artículos tienen de muy buena a regular nivel de calidad.

Hechos relevantes. Consideramos destacables dos cosas: primera, la redacción de los trabajos difiere de la práctica habitual, pues no existe una ordenación clara con títulos y subtítulos, sino una redacción continua, no obstante, aunque no existe 
esa división, en la práctica totalidad se abarcan todos los apartados de una publicación científica, es decir: introducción, objetivos, material y metodología empleada, resultados, discusión de los mismos, conclusiones y bibliografía y, segunda, los Abstracts en inglés resultan muy escuetos y apenas dan información introductoria.

\section{CONCLUSIONES}

Por todo ello concluimos que la revista VKLF tiene, a nuestro juicio, buen grado de recomendación y de calidad científica, aunque mejorable en algunos puntos.

Consideramos que es de gran importancia para los médicos dedicados a la Hidrología Médica el poder acceder a la información de dicha revista. Será más accesible cuando sea editada en inglés.

\section{Agradecimientos}

Nuestro agradecimiento a $\mathrm{M}^{\mathrm{a}}$ Teresa Díaz Berenguer y a Tetyana Tkachenko por el esfuerzo realizado para la traducción de los artículos y a $\mathrm{M}^{\mathrm{a}}$ José Valdemoro, responsable del Servicio Interbibliotecario de la Facultad de Medicina de la Universidad Complutense de Madrid por su inestimable ayuda.

\section{BIBLIOGRAFIA}

1. Maraver F, Morer C. Investigación: PubMed y Termalismo. In: Maraver F, Armijo F. Vademécum II de aguas mineromedicinales españolas. Madrid: Universidad Complutense; 2010. p. 31-42.

2. BioMed Central. Qualitative research review guidelines: RATS. Disponible en: $\mathrm{http}: / /$ www.biomedcentral.com/info/ifora/rats.

3. Begg C, Cho M, Eastwood S, Horton R, Moher D, Olkin I et al. Improving the quality of reporting of randomized controlled trials. The CONSORT statement. JAMA 1996;276(8): 637-9.

4. Jovell AJ, Navarro-Rubio MD. Evaluación de la evidencia científica. Med Clin (Barc) 1995;105(19): 740-3.

5. CEBM. What is EBM?. Oxford: Centre for Evidence-Based Medicine. Disponible en: http://www.cebm.net/ 